\title{
Third Degree Perineal Laceration
}

National Cancer Institute

\section{Source}

National Cancer Institute. Third Degree Perineal Laceration. NCI Thesaurus. Code

C114106.

Injury to perineum involving anal sphincter complex. (adapted from reVIT ALize) 